\title{
Structural and Optical Properties of GaN Nanowires Formed on Si(111)
}

\author{
Sangmoon Hana, Ilgyu Choi ${ }^{\mathrm{a}}$, Jihoon Song, Cheul-Ro Lee ${ }^{\mathrm{a}}$, Il-Wook Cho ${ }^{\mathrm{b}}$, Mee-Yi Ryu ${ }^{\mathrm{b}}$, and Jin Soo Kim ${ }^{\mathrm{a}, *}$ \\ ${ }^{a}$ Department of Electronic and Information Materials Engineering, Division of Advanced Materials Engineering, and Research Center \\ of Advanced Materials Development, Chonbuk National University, Jeonju 54896, South Korea \\ ${ }^{b}$ Department of Physics, Kangwon National University, Chuncheon 24341, South Korea
}

Received July 16, 2018; revised September 20, 2018; accepted September 21, 2018

\begin{abstract}
We discuss the structural and optical characteristics of GaN nanowires (NWs) grown on $\mathrm{Si}(111)$ substrates by a plasmaassisted molecular-beam epitaxy. The GaN NWs with high crystal quality were formed by adopting a new growth approach, so called Ga pre-deposition (GaPD) method. In the GaPD, only Ga was supplied without nitrogen flux on a SiN/Si surface, resulting in the formation of Ga droplets. The Ga droplets were used as initial nucleation sites for the growth of GaN NWs. The GaN NWs with the average heights of 60.10 to $214.62 \mathrm{~nm}$ obtained by increasing growth time. The hexagonal-shaped top surfaces and facets were observed from the field-emission electron microscope images of GaN NWs, indicating that the NWs have the wurtzite (WZ) crystal structure. Strong peaks of GaN (0002) corresponding to WZ structures were also observed from double crystal x-ray diffraction rocking curves of the NW samples. At room temperature, free-exciton emissions were observed from GaN NWs with narrow linewidth broadenings, indicating to the formation of high-quality NWs.
\end{abstract}

Keywords: Plasma-assisted molecular-beam epitaxy, GaN, Nanowires, Si substrate

\section{Introduction}

In the past several years, III-V semiconductor nanowires (NWs) have been actively studied in terms of their fundamental physics and potential device applications due to their superior electrical and optical properties [1-3]. The III-nitride materials have been largely studied in the field of optoelectronic devices such as light-emitting devices and photosensors, which can cover deep ultraviolet (UV) to near infra-red (NIR) window by controlling the composition of $(\mathrm{Ga}, \mathrm{In}, \mathrm{Al}) \mathrm{N}$. However, the formation of high-quality $\mathrm{AlGaN}$ with high $\mathrm{Al}$ content to obtain UV wavelengths and InGaN with high In content to get the NIR window, used as active medium for optoelectronic devices, is very difficult due to inherent physical properties of $\mathrm{Al}$ and In [4-6]. Recently, many researches have been actively performed to use nanostructures instead of thin films for wavelength broadening of III-nitride material system. For examples, GaN, AlGaN, and InGaN NWs formed on $\mathrm{Si}$ substrates have been investigated for the applications of light-emitting diodes and photosensors [7, 8]. Theoretically, GaN NWs formed on Si (111) can be used as an active medium for optoelectronic devices due to their high exciton binding energy and relatively fast carrier

*Corresponding author

E-mail: kjinsoo@jbnu.ac.kr mobility $[9,10]$. One of the most well-known approaches for the formation of Si-based GaN NWs is vapor-liquidsolid (VLS) process, using metal catalysts acting as nucleation seeds of NWs [11,12]. However, it is still difficult to form GaN NW with high crystal quality because of the potential diffusion of metal catalysts into GaN NWs, which may result in chemical contamination $[13,14]$. Y. Wang et al. reported that $\mathrm{Au}$ and $\mathrm{Ni}$, which are typically used as catalytic metals for VLS growth of GaN NWs, introduced deep-level traps in the semiconductor band gap, limiting the performances of optoelectronic devices [14]. To reduce the chemical contamination caused by the metallic catalysts, catalyst-free or self-catalyst methods have been used for the formation of GaN NWs on Si using VLS or Volmer-Weber modes [15-17]. However, it is still difficult to form Si-based GaN NWs with high crystal quality because of the large difference in material parameters between $\mathrm{GaN}$ and $\mathrm{Si}$, including lattice constants and thermal expansion coefficients. That is, catalyst-free GaN NWs on Si substrates showed various shapes and had many structural defects $[15,17]$. J. Ristic et al. reported the spontaneous nucleation and growth mechanisms of $\mathrm{GaN}$ nano-columns on $\mathrm{Si}(111)$ substrates, which may also apply to form III-nitride nanostructures [18]. F. Schuster et al. reported the fundamental role of the substrate material, surface orientation, and termination on the nucleation and growth of GaN NW [19]. However, more detailed 
discussion on the initial growth behavior, internal structure, and optical properties of GaN NWs is necessary. From this consideration, first of all, it is required to form GaN NWs with high crystal quality and uniformity.

In this paper, we report structural and optical properties of GaN NWs formed on $\mathrm{Si}(111)$ substrates by a new growth method, Ga pre-deposition (GaPD) technique. In GaPD growth method, Ga droplets are first formed as initial nucleation sites for the successive NWs on Si (111) substrates. The structural and optical characteristics of the GaN NWs were investigated by field-emission scanningelectron microscopy (FE-SEM), double crystal x-ray diffraction (DCXRD), and photoluminescence (PL) spectroscopy.

\section{Experimental details}

GaN NWs were grown on $\mathrm{Si}(111)$ substrates by a plasma-assisted molecular-beam epitaxy (PA-MBE) with a solid Ga source and a N-plasma cell. A N-plasma flux was obtained by applying an RF generator with a frequency of 13.56 MHz to $\mathrm{N}$ gas with a purity of $99.9999 \%$ [20,21]. Before the growth of GaN NWs, native oxide on $\mathrm{Si}(111)$ substrates was removed by subsequent thermal annealing at a substrate temperature of $900^{\circ} \mathrm{C}$ inside a PA-MBE reactor. Only nitrogen $(\mathrm{N})$ plasma source was supplied to a $\mathrm{Si}(111)$ substrate at a temperature of $800{ }^{\circ} \mathrm{C}$ for 10 minutes (nitridation process) to form a thin $\mathrm{SiN}$ layer with the thickness of $27 \mathrm{~nm}$. After the nitridation process, only Ga flux with a beam equivalent pressure $4.62 \times 10^{-8}$ torr was supplied on the $\mathrm{SiN} / \mathrm{Si}(111)$ surface for 6 seconds to form $\mathrm{Ga}$ droplets, working as initial nucleation sites for $\mathrm{GaN}$ NWs. Figure 1(a) schematically shows the formation process of $\mathrm{Ga}$ droplets on the $\mathrm{SiN} / \mathrm{Si}(111)$ surface by supplying only Ga atoms without N-plasm source. When Ga atoms are supplied to the $\mathrm{SiN} / \mathrm{Si}$ surface, the $\mathrm{SiN}$ phase and $\mathrm{Ga}$ atoms bond SiGaN to form nuclei [22]. Thereafter, Ga adatoms migrate to the sub-nucleus to form a nucleus (I). Finally, Ga droplets with sufficient size are formed to allow Volmer-Weber growth of successive NWs [18]. Meijers et al. reported the use of $\mathrm{Ga}$ droplets for the formation of GaN NWs, where the Ga ball-like features were formed due to the reduction of Ga migration by the excess $\mathrm{N}$ atoms [23]. In our approach, the initial nucleation characteristics of GaN NWs were controlled by manipulating shape and size of $\mathrm{Ga}$ features via deposition time and growth interruption. In Fig. 1(b), Ga and N-plasma fluxes were simultaneously supplied to form GaN NWs via the initial nucleation sites of the Ga droplets (II). For the growth of GaN NWs, Ga adatoms migrate towards the top of the NWs because of chemical potential energy (III) [24]. As schematically shown in Fig. 1(b), the GaN NWs with high crystal quality and uniformity can be grown on the SiN/Si surface by the GaPD method. We controlled the diameter, height and morphology of GaN nanostructures by varying the supply time of Ga atoms in the GaPD method, which is different from the previous approaches using Ga droplets $[23,25]$. GaN NWs were grown on the Ga droplets formed on a $\mathrm{Si}(111)$ substrate at a substrate temperature of $915^{\circ} \mathrm{C}$ and a V/III ratio (defined as the flux ratio of $\mathrm{N}$-plasma to $\mathrm{Ga}$ ) of 108 for 90 minutes (NW1 sample). To investigate the growth behaviors depending on the vertical position of GaN NWs, growth time was respectively increased by 1.5 (NW2 sample) and 2 (NW3 sample) times longer than that of the NW1 sample under the same growth temperature and V/III ratio. To analyze the structural properties of the NW samples, FE-SEM (Hitachi Su-70) and DCXRD (MAX-2500) were used. The accelerating voltage for FESEM measurements was set to $10 \mathrm{keV}$. The PL system was used to analyze the optical characteristics of the GaN NWs. A diode-pumped solid-state laser with the wavelength of $266 \mathrm{~nm}$ was used as an excitation source. The luminescent light from the NW samples was detected by a chargecoupled device with an electrically controlled cooling system.

\section{Results and discussion}

Figures 2(a)-(c) show the cross-sectional (left) and planview (right) FE-SEM images of the NW1, NW2, and NW3 samples, respectively. The average heights (diameters) of the NW1, NW2, and NW3 were measured as 60.10 (43.62),

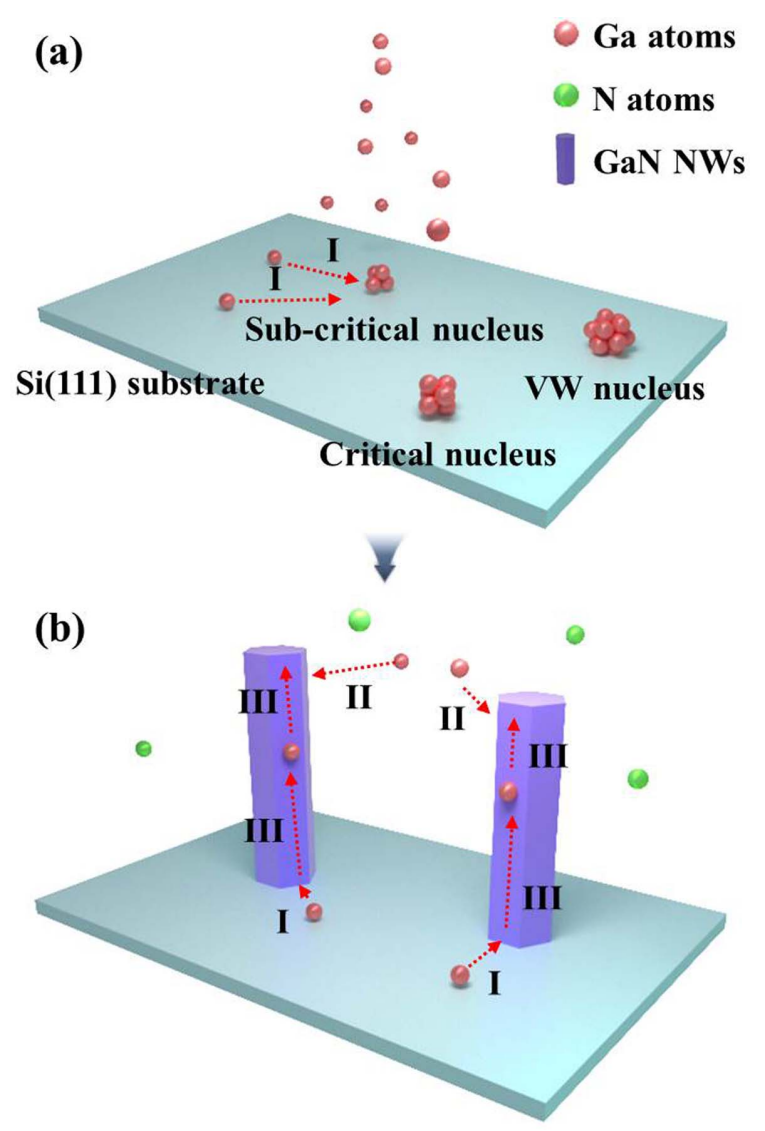

Figure 1. (a), (b) Schematic illustration for the formation of GaN NWs by using GaPD. 
157.01 (65.48), and $214.62 \mathrm{~nm}(65.71 \mathrm{~nm})$, respectively. The top surfaces of the plan-view FE-SEM images of GaN NWs in Fig. 2 clearly show the hexagonal shapes, which are evidences for the formation of the wurtzite (WZ) crystal structure. The inset of each plan-view image is a three-dimensional one for GaN NWs, where the hexagonal facets are clearly observed. This also indicates that the GaN NWs have the WZ crystal structure.

Figure 3 shows the DCXRD of the NW samples grown on $\mathrm{Si}(111)$ substrates. The intensity of the DCXRD rocking curve of the GaN NWs is relatively stronger than that of the $\mathrm{Si}(111)$ substrate. This is related to the $\mathrm{SiN}$ layer formed during nitridation process. That is, the x-ray hardly passes through the SiN layer to Si [26]. The DCXRD peaks are in good agreement with the Joint Committee for
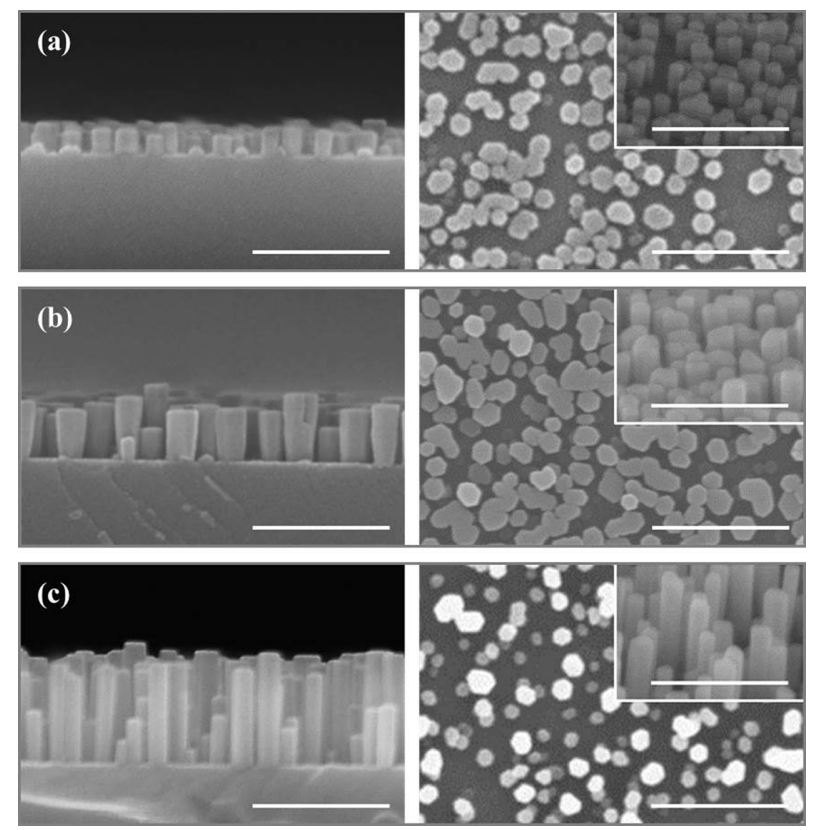

Figure 2. Cross-sectional (left) and plan-view (right) FE-SEM images for the (a) NW1, (b) NW2, and (c) NW3 samples, where the insets are the three-dimensional views. (Scale bar: $300 \mathrm{~nm})$.

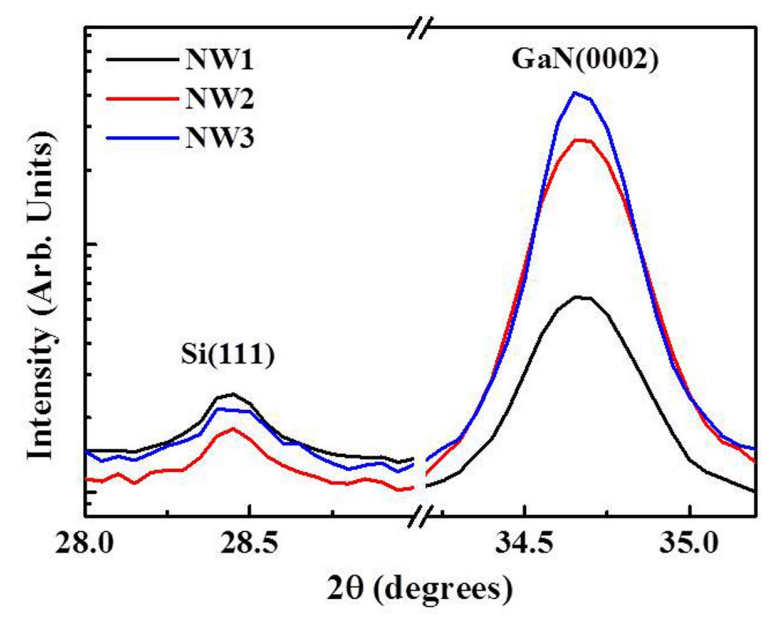

Figure 3. DCXRD rocking curves of the NW samples grown on $\mathrm{Si}(111)$ substrates.
Powder Diffraction Standards (JCPDS) data for the $\mathrm{GaN}$ in the WZ structure and the Si in the cubic structure [27]. That is, the DCXRD peaks corresponding to $\mathrm{GaN}(0002)$ planes for the NW samples are located at $34.67^{\circ}, 34.66^{\circ}$ and $34.65^{\circ}$ for the NW1, NW2, and NW3 samples, respectively, demonstrating that the GaN NWs can be indexed to the WZ crystal structure. The inset is the magnified DCXRD curves for the $\mathrm{GaN}(0002)$ phase, where the peak positions for the NWs were slightly decreased with increasing the average height of the NWs. This is related to the reduction in tensile strain, caused by the large lattice mismatch between $\mathrm{GaN}$ and $\mathrm{Si}$, with increasing the height of the NWs [28]. The peak intensity of the DCXRD rocking curves increases with increasing height of the GaN NWs. This is because as the height of the GaN NWs becomes longer, the amount of the $\mathrm{WZ} \mathrm{GaN}$ structure increases. The full width at half maximum (FWHM) was measured as $0.30,0.25$ and $0.20^{\circ}$ for the NW1, NW2, and NW3 samples, respectively. The FWHM is very narrow compared to those of the previous works $[29,30]$, which indicates that the crystal quality of the GaN NWs in this work is relatively better.

Figures 4(a) and 4(b) show the PL spectra of the GaN NW samples, measured at 10 and $300 \mathrm{~K}$, respectively, at which the excitation power was $3 \mathrm{~mW} / \mathrm{cm}^{2}$. The free-
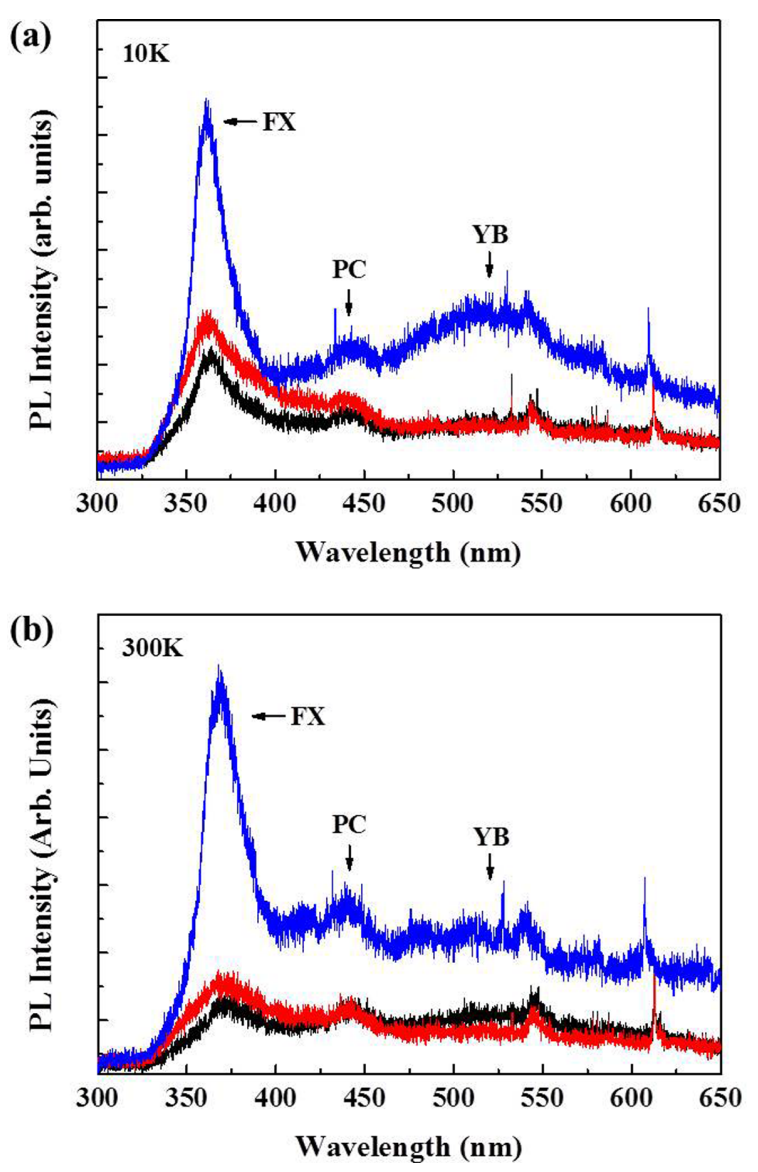

Figure 4. PL spectra for the GaN NW samples measured at (a) 10 and (b) $300 \mathrm{~K}$. 
exciton (FX) peaks in the PL spectra of all the NW samples were clearly observed. Typically, it is very difficult to observe the FX peaks from GaN NWs, largely due to insufficient radiative recombination of carriers inside the NWs over the non-radiative recombination caused by defects and surface states [31]. In Fig. 4(a), the FX peaks were measured as $363.31,361.20$ and $361.20 \mathrm{~nm}$ for the NW1, NW2, and NW3 samples, respectively. The FX peaks of the NW2 and NW3 sample were slightly blueshifted compared to that of the NW1 sample. If we only consider the diameters and heights of NWs, the NW1 sample seems to emit the shortest wavelength among the samples. However, considering exciton Bohr radius and structural dimensions of the NW samples, size quantization effect may be estimated to have very little effect on the emission wavelength $[32,33]$. Therefore, the blue-shift in the emission wavelength for the NW2 and NW3 samples with respect to the NW1 sample can be explained by reduction in strain, which was confirmed at the DCXRD rocking curves. The intensities of the FX peaks for the NW2 and NW3 samples are 1.01 and 1.05 times stronger than that of the NW1 sample, respectively. This is attributed to the improvement in internal crystal quality and the increase in effective volume for the NW2 and NW3 samples, which were already confirmed from the FE-SEM images and DCXRD rocking curves. For the NW samples, a broad yellow-band (YB) peak is weakly observed from 473 to $564 \mathrm{~nm}$, which are related to defects $[9,34,35]$. Also, there is a peak around $434 \mathrm{~nm}$ at the PL spectra measured at 10 and $300 \mathrm{~K}$, which is originated from the polycrystalline (PC) GaN [34,36]. The intensity of YB (PC) peak for the NW3 sample is 1.09 (1.20) and 1.10
(1.17) times stronger than those of the NW1 and NW2 samples, respectively. This is attributed to the increase in the effective number of defects and the PC-GaN with increasing the total volume of $\mathrm{GaN}$. However, the peak intensity ratio of FX to YB for the NW3 sample was measured as 1.16 , which is relatively higher than those of the NW1 (1.05) and NW2 (1.08) samples. Also, the peak intensity ratio of FX to $\mathrm{PC}$ shows the same tendency. These results are related to the increase in the effective volume of GaN NWs with high crystal quality contributing to light emission and the reduction in the amount ratio of defects and PCs with respect to total crystal volume. Once again, the PL results are consistent with the discussion on the DCXRD rocking curves. In Fig. 4(b), the FX peaks were measured as $371.45,367.90$ and $367.89 \mathrm{~nm}$ for the NW1, NW2, and NW3 samples, respectively. The FWHMs at the FX peaks in the PL spectra, corresponding to the size uniformity of GaN NWs, were measured as $52.71,37.72$, $28.10 \mathrm{~nm}$ for the NW1, NW2, and NW3 samples. The FWHM for the NW3 sample is much narrower than those of the NW1 and NW2 samples, indicating that the size uniformity of GaN NWs is relatively better.

Figures 5(a)-(c) show temperature dependence of the PL spectra of the NW samples, measured at the temperature ranging from 10 to $300 \mathrm{~K}$. The emission wavelengths of the NW1, NW2, and NW3 samples were red-shifted with increasing temperature, respectively. The red-shift with temperature is ascribed by the well-known dilation of lattice and the electron-lattice interaction. Figure 5(d) shows summary on the FX peaks and FWHMs of the NW samples with respect to temperature. The FX peaks of the NW1, NW2, and NW3 samples at $300 \mathrm{~K}$ were red-shifted (a)

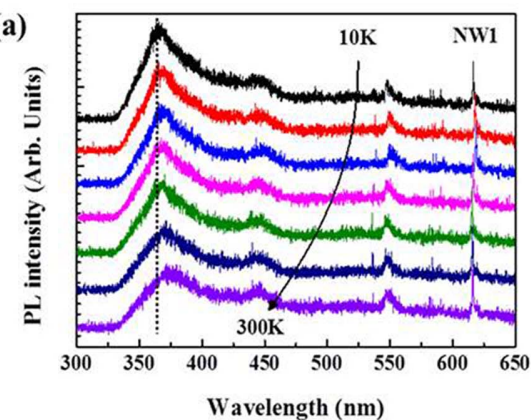

(c)

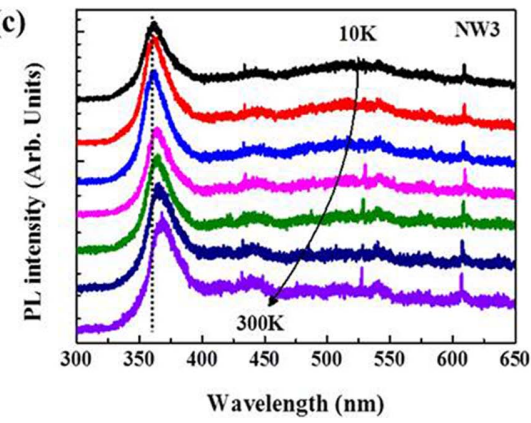

(b)
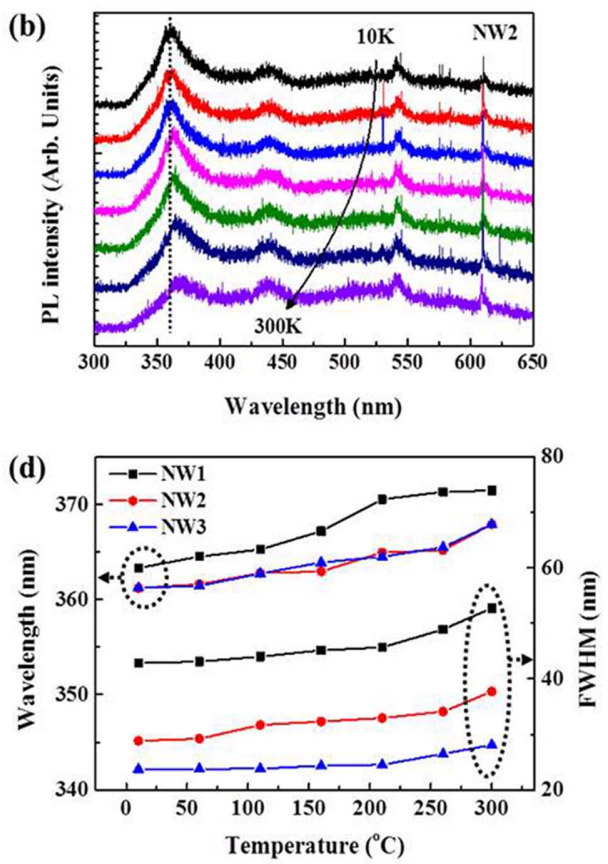

Figure 5. Temperature dependence of PL spectra for the (a) NW1, (b) NW2, and (c) NW3 samples measured in temperature ranging from 10 to $300 \mathrm{~K}$. 
by $8.14,6.70$, and $6.69 \mathrm{~nm}$ from those measured at $10 \mathrm{~K}$, respectively. At the same time, the FWHMs of the NW samples are gradually increased with increasing temperature. The difference in the FWHM of the FX peak between 10 and $300 \mathrm{~K}$ is calculated as $4.39 \mathrm{~nm}$ for the NW3 sample, which is relatively smaller than those of the NW1 (9.86) and NW2 (8.89 nm) samples. This can be explained by the fact that the size distribution of the GaN NWs for the NW3 sample is more uniform than those of the NW2 and NW3 samples. As shown in the FE-SEM images of Fig. 2, since the GaN NWs were closely packed, the carriers thermally activated from certain small NWs by external temperature could be trapped other large NWs [37-39]. For the NW3 sample with better size uniformity compared to the NW1 and NW2 sample, the thermal activation and recapture process of carriers should be relatively small. As a result, the variation in the FWHMs with respect to temperature is relatively small for the NW3 sample compared to the other NW samples.

\section{Conclusions}

In conclusions, GaN NWs with high crystal quality and uniformity were successfully grown on $\mathrm{Si}(111)$ by using newly-suggested GaPD method in a PA-MBE. In the FESEM images for GaN NWs, lateral size and height of GaN NWs were significantly varied with respect to growth time. The DCXRD peaks indicated that the portion of the WZ crystal structure inside the NWs was significantly increased with increasing the height of the GaN NWs. At room temperature, the strong FX emissions were observed from GaN NWs with narrow FWHMs indicating that the highcrystal quality NW were formed by controlling the initial growth behaviors of $\mathrm{Ga}$ atoms using pre-deposited $\mathrm{Ga}$ droplets.

\section{References}

[1] A. C. Ford, J. C. Ho, Y. L. Chueh, Y. C. Tseng, Z. Y. Fan, J. Guo, J. Bokor, and A. Javey, Nano Lett. 9, 360 (2009).

[2] P. Offermans, M. Crego-Calama, and S. H. Brongersma, Nano Lett. 10,2412 (2010).

[3] S. Han, I. Choi, K. Lee, C. R. Lee, S. K. Lee, J. Hwang, D. C. Chung, and J. S. Kim, J. Electron. Mater. 47, 944 (2018).

[4] K. Lee, C. R. Lee, T. H. Chung, J. Park, J. Y. Leem, K. U. Jeong, and J. S. Kim, J. Cryst. Growth, 464, 138 (2017).

[5] K. Lee, C. R. Lee, J. H. Lee, T. H. Chung, M. Y. Ryu, K. U. Jeong, J. Y. Leem, and J. S. Kim, Opt. Express, 24, 7743 (2016).

[6] S. Nikzad, M. Hoenk, A. D. Jewell, J. J. Hennessy, A. G. Carver, T. J. Jones, T. M. Goodsall, E. T. Hamden, P. Suvarna, J. Bulmer, F. Shahedipour-Sandvik, E. Charbon, P. Padmanabhan, B. Hancock, and L. D. Bell, Sensors-Basel. 16, 927 (2016).

[7] J. H. Lee, W. W. Yang, D. W. Chang, S. S. Kwon, and W. I. Park, ACS Appl. Mater. Interfaces 10, 14170 (2018).

[8] S. Deshpande, T. Frost, L. F. Yan, S. Jahangir, A. Hazari, X. H. Liu, J. Mirecki-Millunchick, Z. T. Mi, and P. Bhattacharya, Nano Lett.
$15,1647(2015)$

[9] S. H. Park, W. P. Hong, and J. J. Kim, Superlattice Microst. 109, 254 (2017).

[10] J. G. Rojas-Briseno, G. L. Miranda-Pedraza, and J. C. MartinezOrozco, Phys. Status Solidi B 254, 1 (2017).

[11] C. B. Maliakkal, N. Hatui, R. D. Bapat, B. A. Chalke, A. A. Rahman, and A. Bhattacharya, Nano Lett. 16, 7632 (2016).

[12] T. R. Kuykendall, M. V. P. Altoe, D. F. Ogletree, and S. Aloni, Nano Lett. 14, 6767 (2014).

[13] D. W. Park, S. G. Jeon, C. R. Lee, S. J. Lee, J. Y. Song, J. O. Kim, S. K. Noh, J. Y. Leem, and J. S. Kim, Sci. Rep. 5, 16652 (2015).

[14] Y. W. Wang, V. Schmidt, S. Senz, and U. Gosele, Nat. Nanotechnol. 1,186 (2006).

[15] S. Eftychis, J. Kruse, T. Koukoula, T. Kehagias, P. Komninou, A. Adikimenakis, K. Tsagaraki, M. Androulidaki, P. Tzanetakis, E. Iliopoulos, and A. Georgakilas, J. Cryst. Growth 442, 8 (2016).

[16] S. Fernandez-Garrido, V. M. Kaganer, K. K. Sabelfeld, T. Gotschke, J. Grandal, E. Calleja, L. Geelhaar, and O. Brandt, Nano Lett. 13, 3274 (2013).

[17] R. S. Chen, H. Y. Tsai, C. H. Chan, Y. S. Huang, Y. T. Chen, K. H. Chen, and L. C. Chen, J. Electron. Mater. 44, 177 (2015).

[18] J. Ristic, E. Calleja, S. Fernandez-Garrido, L. Cerutti, A. Trampert, U. Jahn, and K. H. Ploog, J. Cryst. Growth, 310, 4035 (2008).

[19] F. Schuster, S. Weiszer, M. Hetzl, A. Winnerl, J. A. Garrido, and M. Stutzmann, J. Appl. Phys. 116, 044307 (2014).

[20] B. J. Godejohann, E. Ture, S. Muller, M. Prescher, L. Kirste, R. Aidam, V. Polyakov, P. Bruckner, S. Breuer, K. Kohler, R. Quay, and O. Ambacher, Phys. Status Solidi B 254, 1600715 (2017).

[21] F. L. Gao, L. Wen, Z. Z. Xu, J. L. Han, Y. F. Yu, S. G. Zhang, and G. Q. Li, Small 13, 1603775 (2017).

[22] C. K. Xu, M. Kim, S. Y. Chung, J. Chun, and D. E. Kim, Chem. Phys. Lett. 398, 264 (2004).

[23] R. Meijers, T. Richter, R. Calarco, T. Stoica, H. P. Bochem, M. Marso, and H. Lüth, J. Cryst. Growth 289, 381-386 (2006).

[24] V. Consonni, A. Trampert, L. Geelhaar, and H. Riechert, Appl. Phys. Lett. 99, 033102 (2011)

[25] R. K. Debnath, R. Meijers, T. Richter, T. Stoica, R. Calarco, and H. Lüth, Appl. Phys. Lett. 90, 123117 (2007).

[26] S. K. Wu, J. J. Su, and J. Y. Wang, Philos. Mag. 84, 1209 (2004).

[27] G. Santana, O. de Melo, J. Aguilar-Hernandez, R. Mendoza-Perez, B. M. Monroy, A. Escamilla-Esquivel, M. Lopez-Lopez, F. de Moure, L. A. Hernandez, and G. Contreras-Puente, Materials 6, 1050-1060 (2013)

[28] V. M. Kaganer, B. Jenichen, M. Ramsteiner, U. Jahn, C. Hauswald, F. Grosse, S. Fernandez-Garrido, and O. Brandt, J. Phys. D: Appl. Phys. 48, 385105 (2015).

[29] L. Dai, S. F. Liu, L. P. You, J. C. Zhang, and G. G. Qin, J. Phys. Condens. Matter 17, L445 (2005).

[30] V. P. Kladko, A. V. Kuchuk, H. V. Stanchu, N. V. Safriuk, A. E. Belyaev, A. Wierzbicka, M. Sobanska, K. Klosek, and Z. R. Zytkiewicz, J. Cryst. Growth 401, 347 (2014).

[31] T. Auzelle, B. Haas, M. Den Hertog, J. L. Rouviere, B. Daudin, and B. Gayral, Appl. Phys. Lett. 107, 051904 (2015).

[32] C. G. Bailey, S. M. Hubbard, D. V. Forbes, and R. P. Raffaelle, Appl. Phys. Lett. 95, 203110 (2009).

[33] B. T. Liu, S. K. Guo, P. Ma, J. X. Wang, and J. M. Li, Chinese Phys. Lett. 34, 048101 (2017).

[34] J. Y. Li, X. L. Chen, Z. Y. Qiao, Y. G. Cao, and Y. C. Lan, J. Mater. Sci. Lett. 20, 757 (2001).

[35] M. Matys and B. Adamowicz, J. Appl. Phys. 121, 065104 (2017).

[36] A. H. Chin, T. S. Ahn, H. W. Li, S. Vaddiraju, C. J. Bardeen, C. Z. Ning, and M. K. Sunkara, Nano Lett. 7, 626 (2007).

[37] O. Demichel, M. Heiss, J. Bleuse, H. Mariette, and A. F. I. Morral, Appl. Phys. Lett. 97, 201907 (2010).

[38] I. Shalish, H. Temkin, and V. Narayanamurti, Phys. Rev. B 69, 245401 (2004).

[39] P. C. Upadhya, Q. M. Li, G. T. Wang, A. J. Fischer, A. J. Taylor, and R. P. Prasankumar, Semicond. Sci. Technol. 25, 024017 (2010). 